Ana Maria Campos Marques 1 Rivaldo Venâncio da Cunha 2

\section{A medicação assistida e os índices de cura de tuberculose e de abandono de tratamento na população indígena Guaraní-Kaiwá no Município de Dourados, Mato Grosso do Sul, Brasil}

\author{
Assisted treatment and tuberculosis cure \\ and treatment dropout rates in the Guaraní-Kaiwá \\ Indian nation in the municipality of Dourados, \\ Mato Grosso do Sul, Brazil
}

1 Departamento de Tecnologia de Alimentos, Centro de Ciências Biológicas e da Saúde, Universidade Federal de Mato Grosso do Sul. Rua Enramada 39, Campo Grande, $M S$ 79022-450, Brasil. dra.ana@uol.com.br 2 Departamento de Clínica Médica, Universidade Federal de Mato Grosso do Sul. Rua Dr. Armando da Cunha 483, Campo Grande, MS 79051-040, Brasil. rivaldo_venancio@uol.com.br

\begin{abstract}
In January 1998, home treatment regimens were launched in the municipality of Dourados, State of Mato Grosso do Sul, Brazil, and accompanied by indigenous health agents as a means of replacing the previous regimen, in which Guarani-Kaiwá tuberculosis patients were systematically hospitalized for up to six months in the Porta da Esperança Hospital. In order to verify whether this change in strategy had any effect on the cure and treatment dropout rates,, a retrospective study was conducted on 594 patient records from January 1996 to December 1999. Patients were divided into two groups: Group I, treated by hospitalization (291 cases) and Group II, treated as outpatients (303 cases). Group II patients showed a significant increase in the cure rate and a significant reduction in the treatment dropout rate. The study also showed a high tuberculosis prevalence rate in children (40\%), subsequently reported to the competent health authorities and thus launching specific projects to deal with this epidemiological reality. Based on these results, it is recommended that the assisted treatment strategy be adopted for other Indian populations.
\end{abstract}

Key words Tuberculosis; South American Indians; Residential Treatment

Resumo A partir de janeiro de 1998, no Município de Dourados, Estado de Mato Grosso do Sul, Brasil, foi instituído o regime de tratamento domiciliar assistido por agentes indígenas de saúde, em substituição ao regime anterior, quando os pacientes tuberculosos da etnia Guaraní-Kaiwá eram sistematicamente internados para tratamento no Hospital Porta da Esperança, por períodos de até seis meses. Com o objetivo de verificar se a mudança de estratégia promoveu impacto nas taxas de cura e de abandono de tratamento, foram estudados, retrospectivamente, os prontuários de 594 pacientes tratados no período de janeiro de 1996 a dezembro de 1999. Os pacientes foram divididos em dois grupos. Constituíram o Grupo I os casos tratados em regime hospitalar (291 casos) e o Grupo II, os tratados em regime ambulatorial assistido (303 casos). Houve diminuição significativa da taxa de abandono e aumento também significativo da taxa de cura dos pacientes do Grupo II em relação ao Grupo I. Constatou-se uma elevada prevalência de tuberculose (40\%) em menores de 15 anos de idade, o que permitiu alertar as autoridades responsáveis e desencadear projetos específicos para atender a essa realidade epidemiológica. A estratégia de tratamento assistido deveria ser considerada preferencial em outras populações indígenas. Palavras-chave Tuberculose; Índios Sul-Americanos; Tratamento Domiciliar 


\section{Introdução}

A tuberculose, doença infecto-contagiosa, acomete o homem há milênios, como demonstram os esqueletos fósseis de seres humanos com lesões ósseas compatíveis com essa enfermidade encontrados em várias regiões e datados até de 5 mil a.C.

Hoje, considera-se que são responsáveis pelo aumento da endemia tuberculosa no mundo a infecção pelo HIV, o uso de drogas injetáveis, o aumento da pobreza, o aumento do número de desabrigados, a migração, a má nutrição, a urbanização e a perda da qualidade dos programas de controle da tuberculose (Narain et al., 1992; Natal, 1998).

No mundo, são registrados cerca de 8 milhões de casos novos de tuberculose por ano (OPS, 2000). O Brasil concorre com 90 mil casos e o Estado de Mato Grosso do Sul notifica em média mil casos por ano, o que representa uma taxa de incidência de 49/100 mil habitantes (MS, 1997).

A população indígena no Brasil está estimada em aproximadamente 350 mil indivíduos, com maiores concentrações nos Estados do Amazonas, Mato Grosso do Sul, Mato Grosso e Roraima (MS, 2000).

A progressão da integração dos povos indígenas à sociedade nacional faz com que a incidência da tuberculose entre os índios seja significativamente maior do que a encontrada na população não índia (Amarante \& Costa, 2000). De fato, a população indígena do Estado de Mato Grosso do Sul contribui com cerca de $30 \%$ dos casos de tuberculose, sendo que a etnia com maior incidência é a dos Guaraní-Kaiwá, moradores da região sul do Estado, com uma população estimada de 22.600 indivíduos e 160 casos por ano em média, o que significa uma taxa de incidência anual de 700 casos de tuberculose/100 mil habitantes.

As características culturais dos indígenas exigem que o tratamento da tuberculose nessas populações mereça cuidados especiais, dado o risco elevado de abandono e, conseqüentemente, de aumento das taxas de prevalência da doença e de surgimento de casos de tuberculose multiresistente (TBMR).

Os Kaiwá são atendidos no Hospital Porta da Esperança da Missão Caiuá (HPE), localizado no Município de Dourados, Mato Grosso do Sul, desde 1961. A estratégia de tratamento antituberculose adotada pelo hospital consistia na administração dos medicamentos em regime de internação hospitalar por vários meses, até complementar o tratamento.

Essa estratégia de tratamento oferecida pelo HPE, associada a outros fatores culturais pró- prios da população indígena, era causa de desagregação familiar e sócio-econômica, contribuindo para a ocorrência de elevadas taxas de evasão hospitalar - com conseqüente abandono do tratamento - e para a alta incidência de tuberculose nessa população.

Em 1998, o HPE, em parceria com o Município de Dourados e a FUNASA, adotou a estratégia de medicação domiciliar assistida por agentes indígena de saúde (AIS).

O objetivo geral do estudo foi verificar se a mudança de estratégia no tratamento - de tratamento integral em regime de internação hospitalar para tratamento domiciliar assistido teve algum impacto nas taxas de abandono e de cura da tuberculose entre os índios atendidos pelo HPE.

\section{Metodologia}

Foi realizado um estudo retrospectivo de todos os casos de tuberculose em indígenas da região sul do Estado de Mato Grosso do Sul, que receberam diagnóstico e tratamento no HPE da Missão Caiuá, Dourados, Mato Grosso do Sul, no período de janeiro de 1996 a dezembro de 1999, em regime de internação e/ou assistidos pelos AIS em seu domicílio, sob a supervisão da equipe de saúde do hospital e registrados no livro Registro e Controle do Tratamento dos Casos de Tuberculose, conforme as normas do Programa Nacional de Controle da Tuberculose do Ministério da Saúde (PNCT/MS).

Os pacientes foram divididos em dois grupos: - Grupo I: pacientes que iniciaram o tratamento no período de janeiro de 1996 a dezembro de 1997, tendo como estratégia completar o tratamento em regime de internação hospitalar. - Grupo II: pacientes que iniciaram o tratamento no período de janeiro de 1998 a dezembro de 1999 em regime de internação ou não (hospital) e que, em sua maioria, completaram o tratamento em regime domiciliar ou ambulatorial, assistidos pelos AIS, sob a supervisão da equipe de saúde do HPE.

Foram incluídos todos os casos de tuberculose que receberam alta ao completar o tratamento ou que tiveram alta por abandono, conforme os critérios de cura e abandono definidos no PNCT/MS.

Os dados obtidos tiveram a significância estatística verificada por meio do programa Epi Info versão 6. 04b. Foram aceitos como significativos os resultados com risco inferior a 0,05 , utilizando-se a análise bivariada (qui-quadrado de Mantel-Haenszel). 


\section{Resultados}

No período de janeiro de 1996 a dezembro de 1999, no HPE, foram diagnosticados e tratados 670 pacientes com tuberculose, todos registrados no livro Registro e Controle do Tratamento dos Casos de Tuberculose, permanecendo no estudo um total de 594 casos. Desses, 291 iniciaram o tratamento no período de janeiro de 1996 a dezembro de 1997, em regime hospitalar, sendo incluídos no Grupo I. Os demais 303 casos preenchiam os critérios para inclusão no Grupo II, tendo iniciado o tratamento entre janeiro de 1998 e dezembro de 1999, na maioria dos casos, em regime domiciliar ou ambulatorial, supervisionados diretamente pelos AIS. Em ambos os grupos houve predomínio de pacientes do sexo masculino.

A Tabela 1 mostra a distribuição dos pacientes nos Grupos I e II segundo faixa etária. Nos dois grupos observamos um maior percentual de tuberculose entre os pacientes das faixas etárias de menores de 15 anos e de 20 a 34 anos.

Entre os pacientes do Grupo II, 39 (13\%) iniciaram o tratamento em regime ambulatorial assistidos pelos AIS, 264 (87\%) iniciaram o tratamento em regime hospitalar e, desses, apenas $72(27 \%)$ permaneceram internados para completar o tratamento (Tabela 2).

A Figura 1 ilustra a situação de encerramento nos dois grupos.

Comparando o resultado do tratamento entre os anos de 1996, 1997, 1998 e 1999 (Figura 2), observamos uma diminuição significativa no percentual de abandono do tratamento no ano de 1999 em relação aos anteriores.

Distribuindo os pacientes dos Grupos I e II, que receberam alta por abandono segundo o sexo, verificamos que no Grupo I o abandono por parte dos pacientes de sexo masculino é altamente significativo. Esse predomínio não ocorre no Grupo II.

\section{Discussão}

Os resultados do presente estudo permitem reafirmar que a tuberculose ainda é um agravo importante à saúde da população indígena Guaraní-Kaiwá da região sul do Estado de Mato Grosso do Sul.

Em quatro anos, foram diagnosticados e tratados no HPE 670 casos de tuberculose nos índios da região. Considerando que a média da população anual é de 22.600 indígenas e que todos os casos que ocorreram nessa população receberam diagnóstico e tratamento no HPE,
Tabela 1

Distribuição dos casos por grupo e faixa etária.

\begin{tabular}{lrrrr}
\hline \multirow{2}{*}{ Faixa etária } & \multicolumn{2}{c}{ Grupo I } & \multicolumn{2}{c}{ Grupo II } \\
& $n$ & $\%$ & $n$ & $\%$ \\
\hline$<15$ anos & 111 & 38,0 & 130 & 43,0 \\
$15-19$ anos & 25 & 9,0 & 36 & 12,0 \\
$20-34$ anos & 69 & 24,0 & 64 & 21,0 \\
$35-49$ anos & 34 & 12,0 & 28 & 9,0 \\
$50+$ anos & 52 & 18,0 & 45 & 15,0 \\
Total & 291 & 100,0 & 303 & 100,0 \\
\hline
\end{tabular}

$p=0,3408$

Tabela 2

Distribuição do número e percentual de casos

do Grupo II segundo estratégia de administração

do tratamento.

\begin{tabular}{lrr}
\hline Estratégia & Total & $\%$ \\
\hline Agentes indígenas de saúde & 231 & 76,0 \\
Hospital & 72 & 24,0 \\
Total & 303 & 100,0 \\
\hline
\end{tabular}

pode-se inferir uma taxa anual de incidência de todas as formas em torno de 740 casos por 100 mil habitantes. Assim, apesar da existência, desde 1961, de atendimento médico regular com um hospital especializado no diagnóstico e tratamento da tuberculose (HPE), a taxa de incidência permaneceu nos mesmos patamares de 1967 (Czeresnia, 1987), apontando para um baixo rendimento das ações de controle da doença.

O quadro atual da tuberculose entre os índios dessa região não difere em muito da situação dos indígenas de outras regiões do País, onde as taxas de incidência são semelhantes às encontradas na população por nós avaliada (Amarante \& Costa, 2001; Baruzzi et al., 2001; Buchillet \& Gazin, 1998; Escobar et al., 2001).

A distribuição da tuberculose por sexo mantém uma relação entre sexos masculino e feminino de 1,6:1, semelhante à da população geral (Beltran et al., 1983; MS, 1993; Natal et al., 1999).

Na distribuição da variável faixa etária (Tabela 1), não houve diferença entre os Grupos I e II. No entanto, encontramos um percentual elevado de casos de tuberculose em menores de 15 anos (40\%) e na faixa de 20 a 30 anos 
Figura 1

Distribuição do número e percentual de casos de tuberculose nos Grupos I e II segundo a situação de encerramento (cura/abandono).

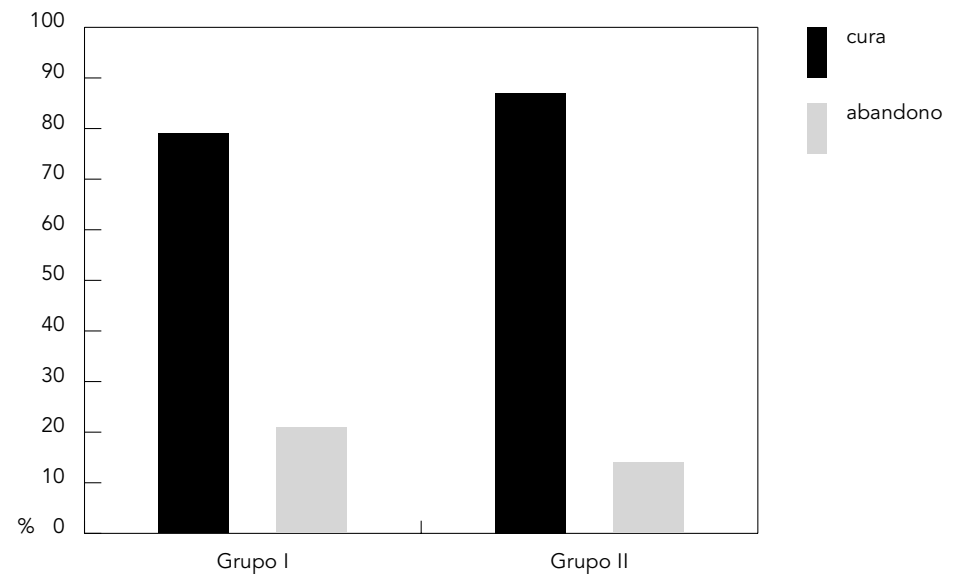

$p=0,0165$

Figura 2

Distribuição do número e percentual de casos por ano segundo resultado de tratamento (cura/abandono).

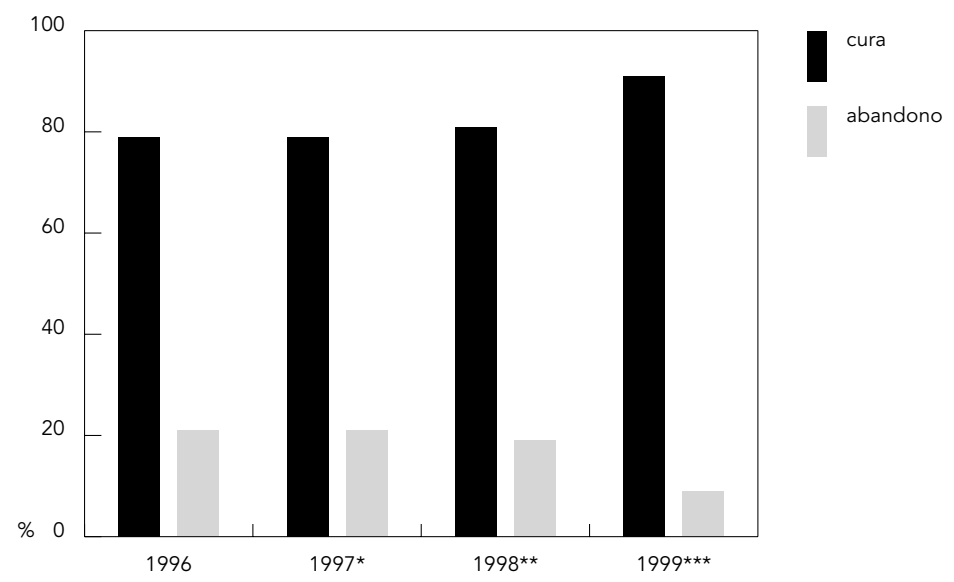

${ }^{\star} p=0,9048 ;{ }^{\star \star} p=0,7616 ;{ }^{* \star *} p=0,0078$
(21\%). Esses números diferem da população geral do Brasil, que apresenta uma concentração maior de casos na faixa etária de 20 a 49 anos, ficando os menores de 15 anos com, em média, 15\% do total de casos (Diniz et al., 1995; MS, 1992; Hijjar, 1992). Além disso, existem evidências de que, na população geral do Brasil, a incidência da tuberculose começa a se deslocar para os idosos, segundo estudo de avaliação da transição etária de incidência e mortalidade da tuberculose no período de 1986 a 1996 (Chaimowicz, 2001).

O deslocamento da curva de incidência de tuberculose para as faixas etárias mais jovens e para as crianças, conforme observado neste estudo, deve ser visto como um evento sentinela em saúde coletiva. Isso denota que está ocorrendo infecção recente por contato com tuberculosos bacilíferos, evidenciando que o controle de contatos não está sendo eficaz (Alland et al., 1994; Alves et al., 2000; Natal, 2000).

Esse processo, na verdade, ocorre desde o início da colonização do Brasil e ainda é observado em alguns grupos de índios isolados, que, ao entrar em contato com a população não índia, são infectados pelo bacilo de Koch. Um caso recente, em que a epidemia de tuberculose acometeu predominantemente menores de 15 anos, ocorreu com a etnia Yanomámi (Buchillet \& Gazin, 1998). No entanto, em relação à população indígena em estudo, o contato com o bacilo é antigo, pois apresenta padrão endêmico alto desde de 1961. Portanto, devemos buscar outras razões, destacando-se:

a) Historicamente, uma taxa elevada de abandono de tratamento ( $>20 \%$ ) nos últimos 40 anos; b) Diagnóstico realizado apenas em função da demanda, sem busca ativa;

c) Omissão de exame dos sintomáticos respiratórios como rotina;

d) Ausência de busca do contatos;

e) Tratamento em regime hospitalar como única estratégia, às vezes internando toda a família, independentemente de doença;

f) Baixo retorno de casos de abandono de tratamento.

Fatores que não podem ser esquecidos no perfil da população estudada - e que, na verdade, podem ser os mais importantes - são os relacionados à extrema pobreza em que vivem esses indígenas. Em todo o mundo, o mapa da prevalência tuberculosa se adapta perfeitamente ao mapa da miséria. A desnutrição, deprimindo o mecanismo da imunidade celular, justifica o maior adoecimento. Por si só, as condições de moradia promíscuas, em cômodos únicos e sem ventilação, propiciam uma maior carga infectante, que também condiciona o 
maior adoecimento por primo-infecção ou por reinfecção exógena.

A situação até agora descrita configurava um quadro alarmante em relação ao controle da tuberculose na população indígena da região sul do Estado de Mato Grosso do Sul. Medidas urgentes de controle deveriam ser implementadas, além de diagnóstico e tratamento. Por essa razão, no início de 2000, antes da conclusão do presente estudo, os resultados preliminares foram repassados às instituições responsáveis pelo controle da tuberculose no Estado. Vale citar que algumas providências foram adotadas e hoje existe o Projeto de Implementação do Controle da Tuberculose na População Indígena de Mato Grosso do Sul, cujo objetivo é aumentar a captação de casos e garantir suplementação alimentar a todos os indígenas doentes de tuberculose e seus contatos, respeitando sua cultura alimentar. As ações tiveram início no Município de Dourados, contando com um Plano de Busca Ativa de Contatos de doentes por tuberculose nos últimos dois anos e priorizando os contatos dos casos pulmonares positivos - essencial para prevenir novos adoecimentos, principalmente em crianças, como recomendado nos trabalhos sobre o tema (Chin, 2000; Dye, 2000; Enarson et al., 1978; Kuaban et al., 1996; Murray et al., 1990; OPS, 1998; Rodrigues \& Smith, 1990).

Em relação à variável história de tratamento anterior nos dois grupos, $92 \%$ dos casos foram classificados como tratamento inicial e $8 \%$ como tratamento por recidiva, demonstrando que praticamente não existe retorno dos abandonos ao serviço de saúde, apesar de o índice de abandono nessa população, ao longo de muitos anos, se manter em patamares elevados. As implicações epidemiológicas dessa situação já foram discutidas acima, a respeito dos casos de tuberculose em menores de 15 anos de idade.

Os resultados positivos da nova estratégia terapêutica podem ser observados na Figura 2, notadamente quanto ao ano de 1999, quando o índice de cura foi de $91 \%$ - bem acima da meta do PNCT (85\%) - e a taxa de abandono, que era em média de $20 \%$, caiu para $9 \%$.

Observamos que no Grupo I ocorre uma maior taxa de abandono na população masculina e que essa diferença desaparece totalmente no Grupo II $(p<0,05)$. A variável sexo masculino representando risco de abandono é um dado conhecido na literatura (van der Werf et al., 1990; Chuah, 1991; Ribeiro et al., 2000). Gonçalves et al. (1999), em estudo realizado na cidade de Pelotas (Rio Grande do Sul) sobre adesão à terapêutica de tuberculose na perspectiva do doente, observaram que o momen- to de vida em que cada paciente se encontra e as características sociais imputadas aos gêneros podem afetar diretamente a forma de lidar com a doença e com o tratamento.

No caso da população estudada, a justificativa para o fenômeno observado pode residir nas implicações sócio-econômicas que o confinamento de um chefe de família ocasionava, já discutidas acima. Premido pela necessidade de manter seu papel de provedor, o paciente tenderia a evadir-se e retornar às suas atividades economicamente produtivas.

Em outras palavras, a estratégia de impor a internação hospitalar por um período de seis meses, adotada anteriormente, tinha um objetivo correto, que seria a garantia de um tratamento completo e adequado. Uma de suas conseqüências mais visíveis, porém, era o desastre financeiro, social e familiar que o afastamento do chefe de família provocava - e que freqüentemente era a causa da evasão. A internação trazia também o estigma da doença difundido na comunidade e a perda de postos de trabalho na usina de cana, grande empregadora dos indígenas da região.

Essa política também pode ser interpretada como uma atitude autoritária que tentou solucionar uma dificuldade cultural impondo um confinamento prolongado, desprezando o indígena enquanto ser humano que necessita de tratamento e não de prisão. Dessa forma, estariam sendo violados os princípios éticos de beneficência e de autonomia do paciente. Sob esse prisma, portanto, tal estratégia não deveria ser utilizada, mesmo que proporcionasse um bom resultado terapêutico.

No entanto, é preciso ponderar que o HPE enfrentava uma realidade adversa, atuando de forma isolada, sem nenhum apoio das autoridades de saúde. Tendo assumido voluntariamente a tarefa de tratar os indígenas, o HPE também recebia pacientes tuberculosos de diversos outros municípios. Some-se a isso a situação econômica de extrema miséria da população indígena dessa região e tem-se um quadro que explica a política adotada.

\section{Conclusões}

O presente estudo evidencia que a estratégia de um tratamento completo em regime de internação não atinge os objetivos almejados. Com a indispensável parceria com as autoridades sanitárias do município e a formação de agentes indígenas de saúde, foi possível realizar o tratamento ambulatorial ou domiciliar assistido. A Figura 1 demonstra que essa estratégia propor- 
cionou resultados altamente significativos em relação à maior taxa de cura e à menor taxa de abandono relativamente ao tratamento hospitalar. Assim, este estudo confirma que, na população indígena, o regime de tratamento ambulatorial ou domiciliar assistido apresenta também os bons resultados alcançados na população em geral (Davidson, 1999; Frieden, 1995; Morrone et al., 1999; OMS, 1996).

A justificativa para a persistência de abandono, mesmo no programa de tratamento domiciliar assistido, foge ao escopo deste trabalho, mas sem dúvida deve ser objeto de estudos para aprimoramento da estratégia.

\section{Referências}

ALLAND, D.; KALKUT, G. E.; MOSS, A. R.; McADAM, R. A.; HAHN, J. A.; BOSWORTH, W.; DRUCKER, E. \& BLOOM, B. R., 1994. Transmission of tuberculosis in New York City. New England Journal of Medicine, 330:1710-1716.

ALVES, R.; SANT'ANNA, C. C. \& LA CUNHA, A. J., 2000. Epidemiologia da tuberculose infantil na cidade do Rio de Janeiro, RJ. Revista de Saúde Pública, 34:409-410.

AMARANTE, J. M. \& COSTA, V. L. A., 2000. A tuberculose nas comunidades indígenas brasileiras na virada do século. Boletim de Pneumologia Sanitária, 8:5-12.

BARUZZI, R. G.; BARROS, V. L.; RODRIGUES, D. R.; MEDEIROS-DE-SOUZA, A. C. \& PAGLIARO, H., 2001. Saúde e doença em índios Panará (KreenAkarôre) após vinte e cinco anos de contato com o nosso mundo, com ênfase na ocorrência de tuberculose (Brasil Central). Rio de Janeiro. Cadernos de Saúde Pública, 17:407-412.

BELTRAN, O. R. P.; MOSCA, C. A.; EISELÊ, C. \& CAROSSO, A., 1983. El abandono del tratamiento en tuberculosis. Realidad actual e perspectivas futuras. Revista Argentina de Tuberculosis, Enfermedades Pulmonares y Salud Pública, 44:11-19.

BUCHILlET, D. \& GAZIN, P., 1998. A situação da tuberculose na população indígena do alto rio Negro (Estado do Amazonas, Brasil). Rio de Janeiro. Cadernos de Saúde Pública, 14:181-185.

CHAIMOWICZ, F., 2001. Transição etária da incidência e mortalidade por tuberculose no Brasil. $R e$ vista de Saúde Pública, 35:81-87.

CHIN, D. P., 2000. Disseminação do Mycobacterium tuberculosis em uma comunidade implementando elementos recomendados de controle da tuberculose. JAMA Brasil, 4:3473-3479.

CHUAH, S. Y., 1991. Factors associated with poor patient compliance with anti-tuberculosis therapy in Northwest Pirak, Malaysia. Tubercle, 72:261264.
CZERESNIA, D. C., 1987. Política indigenista e assistência. Noel Nutels e o Serviço de Unidades Sanitárias Aéreas. Cadernos de Saúde Pública, 4:388401.

DAVIDSON, B. L., 1999. Comparação controlada de terapia diretamente observada $\nu s$. terapia autoadministrada para tuberculose ativa nas áreas urbanas dos Estados Unidos. Chest (Edição em Português), 1:72-76.

DINIZ, L. S.; GERHARDT, G.; MIRANDA, J. A. \& MANCEAUA, J., 1995. Efetividade do tratamento de tuberculose em 8 municípios de capitais brasileiras. Boletim de Pneumologia Sanitária, 3:6-18.

DYE, C., 2000. Tuberculosis 2000-2001: Control, but not elimination. Communicable diseases control, prevention \& eradication, World Health Organization, Geneva, Switzerland. International Journal of Tuberculosis and Lung Diseases, 4:146-152.

ENARSON, D. A.; GRZYBOWSKI, S. \& DORKEN, E., 1978. Failure of diagnosis as factor in tuberculosis mortality. CMA Journal, 24:1520-1522.

ESCOBAR, A. L.; COIMBRA Jr., C. E. A.; CAMACHO, L. A. \& PORTELA, M. C., 2001. Tuberculose em populações indígenas de Rondônia, Amazônia, Brasil. Cadernos de Saúde Pública, 17:285-298.

FRIEDEN, T. R.; FUJIWARA, P. I.; WASHKO, R. M. \& HAMBURG, M. A., 1995. Tuberculosis in New York City - Turning the tide. New England Journal of Medicine, 333:229-233.

GONÇALVES, H.; DIAS-DA-COSTA, J. S.; MENEZES, A. M. B.; KNAUTH, D. \& LEAL, O. F., 1999. Adesão à terapêutica da tuberculose em Pelotas, Rio Grande do Sul: Na perspectiva do paciente. Cadernos de Saúde Pública, 15:777-787.

HIJJAR, M. A., 1992. Epidemiologia da tuberculose no Brasil. Informe Epidemiológico do SUS, 1:53.

KUABAN, C.; KOULLA-SHIRO, S.; LEKAMA-ASSIENE, T. \& HAGBE, P., 1996. Despistage de la tuberculose chez les sujets-contacts en 1993 et 1994 à Yaounde, Cameron. Médicine Tropicale, 56:156-158. 
MORRONE, N.; SOLHA, M. S. S.; CRUVINEL, M. C.; MORRONE Jr., N.; FREIRE, J. A. S. \& BARBOSA, Z. L. M., 1999. Tuberculose: Tratamento supervisionado "vs" tratamento auto-administrado. Experiência ambulatorial em instituição filantrópica e revisão de literatura. Jornal de Pneumologia, 25: 198-206.

MS (Ministério da Saúde), 1992. Controle da Tuberculose: Uma Proposta de Integração Ensino Serviço. Rio de Janeiro: MS.

MS (Ministério da Saúde), 1993. Reunião de avaliação operacional e epidemiológica do PNCT, na década de 80. Boletim de Pneumologia Sanitária (Número Especial):9-70.

MS (Ministério da Saúde), 1997. Tuberculose - Distribuição de casos novos notificados em 1996 e 1997 (até o primeiro trimestre), por unidade federada. Informe Epidemiológico do SUS, 6:153.

MS (Ministério da Saúde), 1999. Plano Nacional de Controle da Tuberculose. Brasília: MS.

MS (Ministério da Saúde), 2000. Política Nacional de Atenção à Saúde dos Povos Indígenas. Brasília: MS.

MURRAY, C. J. L.; STYBLO, K. \& ROUILLON, A., 1990. Tuberculosis in developing countries: Burden, intervention and cost. Bulletin of the International Union against Tuberculosis and Lung Diseases, 65:6-24.

NARAIN, J. P.; RAVIGLIONE, M. C. \& KOCHI, A., 1992. HIV - Associated tuberculosis in developing countries: Epidemiology and strategies for prevention - Longman Group UK Ltd. Tubercle and Lung Disease, 73:311-321.

NATAL, S., 1998. A retomada da importância da tuberculose como prioridade para ações de saúde pública. Boletim de Pneumologia Sanitária, 6:40-41.
NATAL, S., 2000.Tuberculose na criança. Boletim de Pneumologia Sanitária, 8:20-25.

NATAL, S.; VALENTE, J.; GERHARDT, G. \& PENNA, M. L., 1999. Modelo de predição para o abandono do tratamento da tuberculose pulmonar. Boletim de Pneumologia Sanitária, 7:65-77.

OMS (Organización Mundial de la Salud), 1996. Grupos em Riesgo. Informe de la OMS sobre la Epidemia de Tuberculosis. Geneva: OMS.

OPS (Organización Panamericana de la Salud), 1998. El control de la tuberculosis en las Américas. Boletín Epidemiológico, 19:1-8.

OPS (Organización Panamericana de la Salud), 2000. Boletín del programa regional de tuberculosis de la Oficina Regional de la Organización Mundial de la Salud (OMS). Tuberculosis, 3:1-7.

RIBEIRO, S. A.; AMADO, V. M.; CARMELIER, A. A.; FERNANDES, M. M. A. \& SCHENKMAN, S., 2000. Estudo caso-controle de indicadores de abandono em doentes com tuberculose. Jornal de Pneumologia, 26:291-296.

RODRIGUES, L. C. \& SMITH, P. G., 1990. Tuberculosis in developing countries and methods for its control. Transactions of the Royal Society of Tropical Medicine and Hygiene, 84:739-744.

van der WERF, T. S.; DADE, G. K. \& van der MARK, T. W., 1990. Patient compliance with tuberculosis treatment in Gana: Factors influencing adherence to therapy in a rural service programme. $\mathrm{Tu}$ bercle, 71:247-252.

Recebido em 11 de junho de 2002

Versão final reapresentada em 10 de dezembro de 2002 Aprovado em 26 de maio de 2003 